\title{
How to Foster Citizens' Statistical Reasoning: Implications for Genetic Counseling
}

\author{
Stephanie Kurzenhäuser Ralph Hertwig \\ Department of Psychology, University of Basel, Basel, Switzerland
}

\section{Key Words}

Risk communication - Statistical reasoning $\cdot$ Diagnostic technologies $\cdot$ Representation of information

\begin{abstract}
Objectives: Our aim is to provide an overview of key research findings from cognitive psychology regarding effective ways of communicating statistical information, and to point out the implications of these findings for genetic testing. Method: We review the literature on the presentation of statistical information in diagnostic test results, discuss various representations that invite misunderstandings, and propose alternative representations that foster understanding. Results: Single-event probabilities, conditional probabilities and relative risks are easily misunderstood. Specifying the class of events to which a probability refers and using natural frequency statements improve understanding. Conclusions: Cognitive psychology has identified simple and effective tools for improving statistical reasoning. They can help to improve the public's understanding of diagnostic test results.
\end{abstract}

Copyright (C) 2006 S. Karger AG, Basel
In 2002, an issue of Wired magazine featured the experiences of a journalist who underwent the 'world's first top-to-bottom gene scan'. Developed as a prototype by a biotech company, this scan screens for a total of 77 genetic disease markers, all of them linked to a person's life expectancy. To his distress, the author learned that he had two mutations that raised his risk of a heart attack:

My face drains of color as the news sink in ... I shouldn't be surprised, since I'm told everyone has some sort of disease-causing mutation. Yet I realize that my decision to take a comprehensive DNA test has been based on the rather ridiculous assumption that I would come out of this with a clean genetic bill of health [1, p. 183].

Puzzled as to how to interpret the screening results, he consulted a genetic counselor who informed him that 'genetic data are seldom clear-cut', meaning that for most diseases, a positive genetic test result does not equal a $100 \%$ chance of disease. But this lack of determinacy did not provide much comfort either, and ultimately, the journalist regretted having subjected himself to this cutting-edge technology: the knowledge of the wicked mutations 'deep inside me will be nagging me every time I get short of breath' [1, p. 187].

Soon the top-to-bottom gene scan will no longer have the ring of science fiction. In fact, hardly a month passes without a new entry in the already impressive list of inherited disorders and risk factors that can be detected

\begin{tabular}{ll}
\hline KARGER & ( ) 2006 S. Karger AG, Basel \\
Fax +41 61306 $1234-2795 / 06 / 0093-0197 \$ 23.50 / 0$ \\
$\begin{array}{l}\text { E-Mail karger@karger.ch } \\
\text { www.karger.com }\end{array}$ & $\begin{array}{l}\text { Accessible online at: } \\
\text { www.karger.com/cmg }\end{array}$
\end{tabular}


through genetic testing. It is widely believed that genetic tests will soon be part of everyday medical care and will be key in detecting vulnerability to disease and to drug response in healthy individuals [2].

\section{More Knowledge, More Certainty?}

More knowledge does not necessarily mean more certainty, as the journalist of Wired magazine discovered with regret. He, like many people, seems to have projected the illusion of certainty onto this new technology - a technology that more than other screening methods for health risks may prompt such an illusion because of its association with the typical image of genetic endowment as something akin to a 'blue-print' or a 'program' [3]. However, many excellent diagnostic technologies (e.g., DNA fingerprinting, HIV tests, and gene scans) do not offer certainty. They merely promise to quantify in statistical terms the chance with which an event has occurred or will occur. Therefore, people need to come to terms with statistical information associated with the results of diagnostic technologies such as gene scans.

But how can people become competent consumers of crucial information rendered available by genetic technologies? A simple answer is by way of more knowledge. Indeed, despite the public's interest in the topic of genetic technologies, most people see themselves as only moderately genetically literate [2]. In addition, genetic counseling practice reveals how difficult it is to reliably remove gaps in clients' knowledge, and to convey risk information such that recipients understand and use it as intended by the counselors [4, 5]. Like the Wired magazine journalist, for instance, many recipients of genetic counseling appear to feel the need to convert statistical information (e.g., 'a $20 \%$ chance') into categorical, definite terms (e.g., high versus low risk), thus getting rid of the probabilistic aspect of the statement [4]. The belief that more knowledge is the panacea to lack of understanding and, ultimately, acceptance of modern technologies also appears to underlie the deficit model of the public's understanding of science. In this model, the frail acceptance of modern technologies is traced to John Sixpack's lack of accurate scientific knowledge about them [6, 7]. Recently, however, the deficit model has been criticized on both empirical and theoretical grounds [6-8], and the debate about public acceptance of genetic technologies has turned to other variables such as the public's lack of trust in science [8].

Without questioning the importance of trust as a prerequisite for the public's acceptance of technologies, we focus on what we view as another key prerequisite, namely, the insight that modern diagnostic technologies are not revelation machines that propel us from the twilight of probability. This insight, in turn, is closely linked with the public's ability to understand and benefit from the statistical information that represents the typical outcomes and foundation of diagnostic tests, for instance information about the accuracy of the test result or about the disease susceptibility that follows.

\section{From Innumeracy to Insight}

At the beginning of the twentieth century, the father of modern science fiction, the writer H.G. Wells, is reported to have predicted that 'statistical thinking will one day be as necessary for efficient citizenship as the ability to read and write'. At the end of the last century, the mathematician Paulos [9] investigated how much - or rather, how little - progress people had made in this respect. In Innumeracy, Paulos tells the story of a weather forecaster on American television who reported that there was a $50 \%$ chance of rain on Saturday and a 50\% chance of rain on Sunday, ergo, he concluded that there was a $100 \%$ chance of rain that weekend.

The inability to reason appropriately about probabilities, however, is not an affliction limited to TV weather forecasters, but is widespread in many Western countries [10]. Statistical innumeracy, we emphasize, is not simply a problem within the individual mind, or a problem of lack of knowledge. The problem lies in the failure to communicate risk in understandable ways that allow a person to draw conclusions or inferences from the risks (e.g., the predictive value of a genetic test result). But how can risk be communicated to foster insight? Our conjecture is that cognitive psychology has identified mind tools that can help laypeople and experts alike to improve their comprehension of statistical information. In what follows, we present representations of statistical information that invite misunderstandings and hamper the ability to draw conclusions from such information. We then propose alternative ways of representing the same statistical information that facilitate people's understanding ${ }^{1}$. These representations work without explicit instruction. They work because they take into account that the cognitive algorithms of the mind are tuned to specific representations of information.

1 We focus on one aspect of representation of statistical information. Others, not discussed here, are the impact of visual presentation [11-13] and framing $[14,15]$ of statistical information. 


\section{Sources of Misunderstandings and How to Remove Them}

\section{Single-Event Probabilities}

To communicate risk in the form of a single-event probability means to make a statement of this type: 'The probability that an event will happen is $\mathrm{x} \%$.' The statement 'Given the genetic test result, your chance of developing breast cancer is $85 \%$ ' is an instance of risk communication in terms of a single-event probability. Such a statement can be confusing, because a single-event probability, by definition, leaves open the class of events to which the probability refers.

The following occurrence illustrates this ambiguity [10]. A psychiatrist prescribed Prozac ${ }^{\circledR}$ to his mildly depressed patients. He would inform them that they had a ' $30-50 \%$ chance of developing a sexual problem' such as impotence or loss of sexual interest. Hearing this, patients were concerned and anxious. After learning about the ambiguity of single-event probabilities, the psychiatrist changed his way of communicating risks. He told patients that out of every 10 people to whom he prescribes Prozac, 3-5 will experience a sexual problem. This way of communicating the risk of side effects seemed to put patients more at ease. The psychiatrist realized that he had never checked how his patients understood what 'a 30-50\% chance of developing a sexual problem' meant. It turned out that many had thought that something would go awry in $30-50 \%$ of their sexual encounters.

The important insight from this doctor-patient interaction is that the psychiatrist's initial approach to risk communication left the reference class unclear: does the $30-50 \%$ chance refer to a class of people (patients who take Prozac), to a class of events (a given person's sexual encounters), or to some other class? Whereas the psychiatrist's reference class was the total number of his patients taking Prozac, his patients' reference class was their own sexual encounters. It was only thanks to a coincidence that the psychiatrist chose a different way to communicate the chances of side effects, thus realizing his patients' previously unnoticed misunderstandings.

When risks are solely communicated in terms of single-event probabilities, people have little choice but to fill in a reference class spontaneously. This was demonstrated in a recent study that asked pedestrians in one American and four major European cities about their understanding of a probabilistic weather forecast (' $30 \%$ chance of rain tomorrow') [16]. Only in New York did a majority of people supply the standard meteorological interpretation, namely, that when the weather conditions are like today, in 3 out of 10 cases there will be (at least a trace of) rain the next day. In each of the European cities, this interpretation was judged as the least appropriate. The preferred interpretation in Europe was that it will rain tomorrow ' $30 \%$ of the time', followed by 'in 30\% of the area'. In other words, even numerical probabilities can be interpreted by members of the public in multiple, possibly contradictory ways.

The ambiguity of a single-event probability and the resulting misunderstandings are not limited to the understanding of the risks of side effects and precipitation. Single-event probabilities can also have far-reaching consequences when they are, for instance, used by expert witnesses to explain DNA evidence in court [17], used by clinical psychologists and psychiatrists to predict the possibility that a mental patient will commit violent acts [18], and used by medical organizations to communicate the benefits and risks of treatments [10].

The way to reduce confusion about what single-event probabilities mean is straightforward: always communicate the reference class to which the single-event probabilities pertain. For instance, ' $30 \%$ probability of rain tomorrow' does not refer to how long, in what area, or how much it will rain. It means that in three out of ten times when meteorologists make this prediction, there will be at least a trace of rain the next day. Alternatively, one may avoid confusion by replacing ambiguous single-event statements with frequentist statements. For instance, the psychiatrist may simply explain to his patients that ' 3 out of every 10 patients have a side effect from this drug' [15].

\section{Conditional Probabilities}

Despite prior experience, patients have often only little knowledge about the accuracy of diagnostic tests [19]. Limited knowledge, in turn, is often tantamount to the belief that the diagnostic tests are more accurate and more predictive than they actually are [20]. Genetic testing in particular is perceived to be extremely predictive, perhaps even infallible [3, 17]. Thus, for many people, the fact that diagnostic tests do not produce certainty comes as a surprise. For instance, in a recent survey in the Netherlands, $32 \%$ of respondents thought that if prenatal tests such as chorionic villus sampling and amniocentesis came out negative, the child would definitely be healthy [2]. Believing that a negative test result means having zero risk is a misunderstanding that abets an illusory sense of certainty. Such a misunderstanding stems in part from the way that the test results are presented [21]. 
When attempting to comprehend results of genetic tests, people need to confront the fact that tests are not failsafe. Tests make correct as well as incorrect identifications, and the chance of each comes, typically, in terms of conditional probabilities. The following statement conveys the sensitivity of a test: 'If a child has a genetic disease, the probability that prenatal test $\mathrm{X}$ turns out positive is $90 \%$.' Such conditional probabilities are in danger of being misunderstood. Specifically, the conditional probability of a positive test result given a genetic disease (the sensitivity of a test) is confused with the inverse probability of genetic disease given a positive test result (the predictive value of a test).

Experts are not immune to this confusion. In one study, experienced doctors read the following information about a screening test for colorectal cancer [22]:

The probability of colorectal cancer in a certain population is $0.3 \%$ [base rate]. If a person has colorectal cancer, the probability that the haemoccult test is positive is $50 \%$ [sensitivity]. If a person does not have colorectal cancer, the probability that he still tests positive is $3 \%$ [false-positive rate].

The doctors were then asked to estimate the probability that someone who tests positive actually has colorectal cancer. The correct estimate for the positive predictive value of the test is 5\%. Yet, answers ranged from 1 to $99 \%$, and every second doctor estimated the probability as $50 \%$ (the sensitivity) or $47 \%$ (the sensitivity minus false-positive rate).

The difficulties that people have in reasoning with conditional probabilities are often presented as if they were the natural consequence of flawed mental software [23]. This view, however, overlooks the fundamental fact that the human mind processes information through external representations, and that selecting representations can improve or impair our understanding of statistical information. Consider the following alternative representation of the above information:

Out of every 10,000 people, 30 have colorectal cancer. Of these 30,15 will have a positive haemoccult test. Out of the remaining 9,970 people without colorectal cancer, 300 will still test positive. How many of those who test positive actually have colorectal cancer?

As before, the correct answer is 5\%, i.e. 15 out of 315 . In responding to this natural frequency representation, 16 out of 24 physicians gave the correct answer. In contrast, only 1 out of 24 physicians could give the correct answer when the statistical information was expressed as probabilities [22].
As figure 1 shows, representation matters because the statistical reasoning that is required, for instance, to calculate the positive predictive value of a test (Bayesian reasoning) is relatively simple with natural frequencies, but becomes cumbersome the moment conditional probabilities are introduced (fig. 1). Studies that previously concluded that physicians [24] and laypeople [25] have great difficulties in understanding the predictive value of test results, typically presented information in terms of probabilities and percentages. Natural frequencies, unlike probabilities and percentages, carry implicit information about base rates, thus reducing the number of computations required to determine the positive predictive value of a test [22]. Natural frequencies also correspond to the way in which humans have experienced statistical information over most of their history [for a discussion of this and alternative explanations, see ref. 26].

Representing risk in terms of natural frequencies fosters statistical reasoning. This finding is robust for laypeople and experts [27-29]. Although the beneficial effect of natural frequencies occurs without providing more knowledge through training or instruction - just by changing the representation of information - people can be explicitly encouraged to translate conditional probabilities into natural frequencies. Tutorials teaching people to change representations rather than having them apply mathematical formulas (the formula needed to solve the diagnostic inference problem mentioned above is Bayes' rule) turn out to be much more effective in improving diagnostic inferences [30, 31].

\section{Relative Risks}

In addition to single-event probabilities and conditional probabilities, there is another source of misunderstandings in communicating risk: relative risks. What is the benefit of mammography screening on the risk of dying from breast cancer? Women who ask this question often hear the following answer: by undergoing mammography screening, women aged over 40 years reduce their risk of dying from breast cancer by $25 \%$. This number is a relative risk reduction, which is the absolute risk reduction divided by the proportion of patients who die without undergoing screening. This number is mute on the underlying raw frequencies. The frequencies for these data are derived from four Swedish randomized trials for women between 40 and 74 years of age [32]. Out of 1,000 women who did not participate in mammography screening, 4 died of breast cancer. Out of 1,000 women who did participate in mammography screening, 3 died of breast cancer. Screening thus saved the life of one out of four 


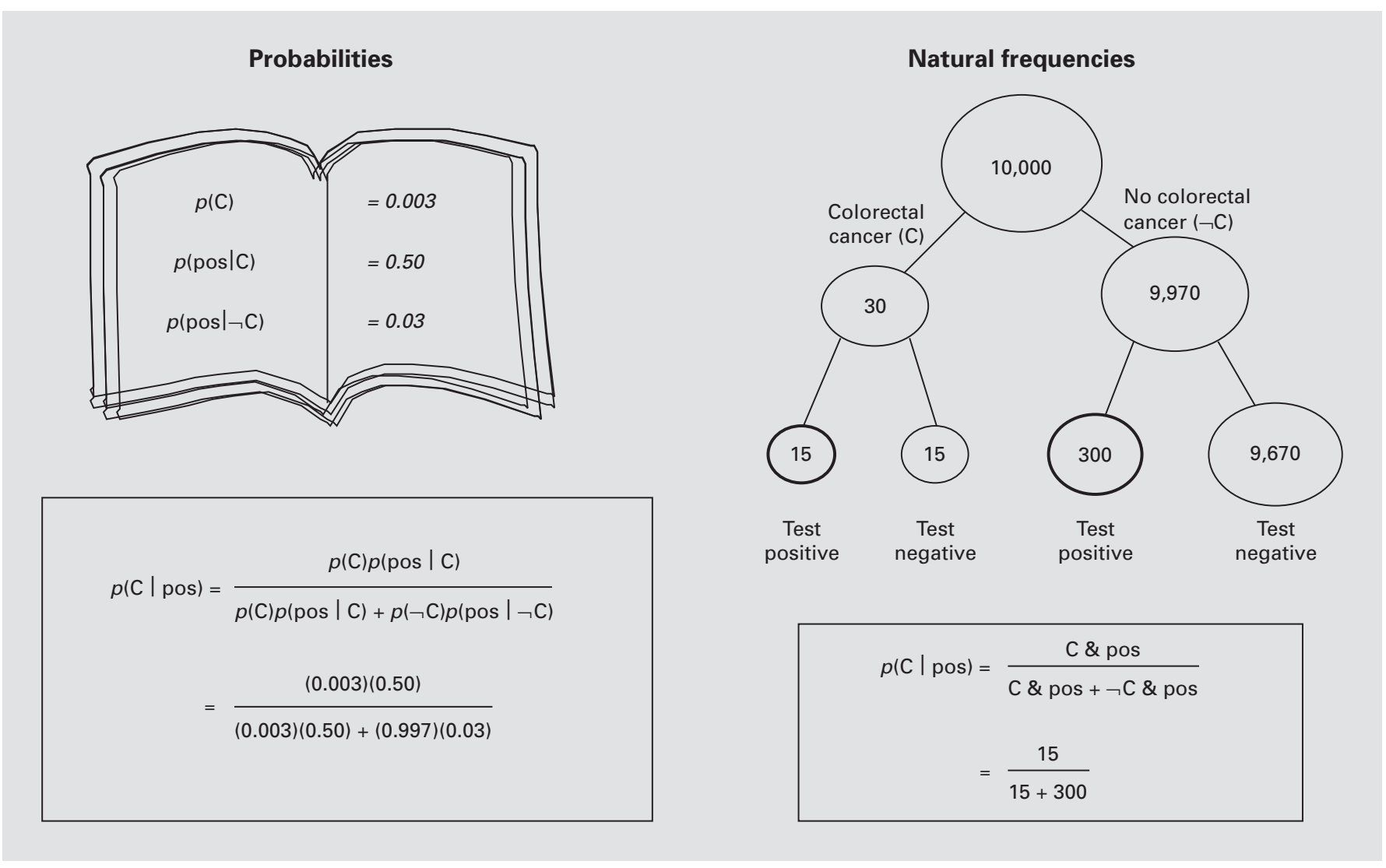

Fig. 1. Explanation of why natural frequencies facilitate the computation of the probability $p(C \mid p o s)$ of cancer given a positive test (a form of Bayesian reasoning). The symbols $C$ and $\neg C$ stand for colorectal cancer and no colorectal cancer, respectively, and pos stands for a positive test result. One can see that Bayes' rule for probabilities involves more calculations than that for natural frequencies.

women who would otherwise have died from breast cancer, which is a reduction of $25 \%$.

Relative risk reduction is not the only way to represent the benefit of mammography. Alternatively, its benefits can be framed in terms of absolute risk reduction, namely, the proportion of women who die from breast cancer without undergoing a mammography screening minus those who die despite being screened. Screening reduces the number of people who die from 4 to 3 in 1,000. That is, the absolute risk reduction is 1 in 1,000 , which is $0.1 \%$. Still another representation of the same information is the number needed to treat, which is the number of people who must participate in the screening to save one life. This number can be derived from the absolute risk reduction. The number of people who needed to be screened to save one life is 1,000 , because the screening prevents 1 in 1,000 deaths.
The relative risk reduction looks more impressive than the absolute risk reduction. Health organizations inform patients about the benefits of mammography screening almost exclusively in terms of the relative risk reduction, and perhaps not surprisingly, people are more likely to prefer an intervention if it is advertised in terms of relative risk reduction rather than in absolute risk reduction $[10,33]$. It has been suggested that people draw wrong conclusions from relative risk reduction. Indeed, the fact that people frequently overestimate the benefits of screening programs [20] is consistent with the possibility that they assume that the relative risk reduction (e.g., 25\%) applies to those who participate in screenings when in fact it refers to the people who die of the disease without having been screened.

All three representations of the raw frequencies are correct. Yet, they do suggest different amounts of benefit, 
and thus, are likely to elicit different expectations. We propose that risk can be communicated more effectively in terms of absolute reduction, rather than in terms of relative risk reduction. At a minimum, both pieces of information should be provided [15].

\section{Statistical Information: Numbers or Words?}

Relative risk reduction, single-event probabilities, and conditional probabilities are frequently used to inform patients about the outcomes and consequences of medical test results. There is strong consensus that health professionals, especially in the case of genetic counseling, are ethically obliged to provide such statistical information, thus enabling patients to make informed decisions [5]. The alternative representations that we have outlined can foster patients' understanding of statistical information.

Notwithstanding the power of alternative numerical presentation, it is sometimes suggested that numerical information should be replaced with simple verbal categorizations of risk ('high' or 'low' risk). Admittedly, enabling people to thoroughly understand numerical expressions of risk is not a trivial task [34], and alternative representations will not invariably succeed. However, numbers appear to be better suited than words for communicating risk. First, verbal quantifiers such as 'high' or 'moderate' are less precise than numbers, thus inviting more varied interpretations and achieving even less accurate understanding [11,21; but see 35 for an exception]. For example, what is 'unlikely' for a counselor might well be 'likely' for the patient [11]. Second, even though most people like to provide information in categorical terms, they prefer to receive information numerically when they have to base a decision on it [36]. For instance, in genetic counseling for breast and ovarian cancer, $73 \%$ of the counselees expressed a preference for the risk to be described in quantitative formats [37]. In addition, a numeric statement of risk can increase trust in and comfort with the risk information, compared with a purely verbal statement [38]. To conclude, even though people often translate numerical into categorical risk information during their decision making process [4], they expect numbers to begin with, and they appear to benefit more from numbers than from words - if represented in the right way. Thus, as H.G. Wells purportedly predicted, statistical thinking is indeed indispensable for efficient citizenship, whether the citizen is a patient, a doctor, a lawyer, or a consumer of the daily weather report.

\section{Conclusions}

All-encompassing genetic screening promises to provide us with previously unimaginable ways of responding to the risks of diseases. Whether this promise will be fulfilled, however, will in part also depend on whether people come to terms with the fact that the new diagnostic tools are not omniscient. They do not produce certainty, but they quantify previously unobservable risks. One step toward overcoming the illusion of certainty is to be able to reason statistically. There is, of course, more to the counseling process than good statistical reasoning on the part of the counselor and the client, respectively [5], but without statistical reasoning, the best technology will be of little value.

Innumeracy, in turn, cannot simply be overcome by trite calls for more knowledge about diagnostic technologies. More information will not automatically produce better-informed citizens and higher levels of public welfare. Instead, information has to be communicated intelligibly. For this reason, new technologies need psychology [28]. Cognitive psychology provides an understanding of how people process statistical information, thus enabling the design of simple but powerful mind tools for improving statistical reasoning. The public need not suffer from the tyranny of too much information, but should benefit from its smart representation.

\section{Acknowledgments}

We would like to thank Laura Wiles for improving our manuscript, and the Jacobs Foundation for their financial support (grant to Ralph Hertwig and Michaela Wänke). 


\section{References}

1 Duncan DE: DNA as destiny. Wired 2002;11: 180-187.

-2 Henneman L, Timmermans DR, van der Wal G: Public experiences, knowledge and expectations about genetics and the use of genetic information. Community Genet 2004;7:33-43.

-3 Davison C, Macintyre S, Smith GD: The potential social impact of predictive genetic testing for susceptibility to common chronic diseases: a review and proposed research agenda. Sociol Health Illn 1994;16:340-371.

-4 Bottorff JL, Ratner PA, Johnson JL, Lovato CY, Joab SA: Communicating cancer risk information: the challenges of uncertainty. Patient Educ Couns 1998;33:67-81.

$\checkmark 5$ Marteau TM, Dormandy E: Facilitating informed choice in prenatal testing: how well are we doing? Am J Med Genet 2001;106:185190.

-6 Bak HJ: Education and public attitudes toward science: implications for the 'deficit model' of education and support for science and technology. Soc Sci Q 2001;82:779-795.

7 Kerr A, Cunningham-Burley S, Amos A: The new genetics and health: mobilizing lay expertise. Public Underst Sci 1998; 7:41-60.

8 Cunningham-Burley $\mathrm{S}$, this issue.

9 Paulos JA: Innumeracy: Mathematical Illiteracy and Its Consequences. New York, Vintage Books, 1988.

10 Gigerenzer G: Reckoning with Risk: Learning to Live with Uncertainty. London, Penguin Books, 2002.

11 Burkell J: What are the chances? Evaluating risk and benefit information in consumer health materials. J Med Libr Assoc 2004;92: 200-208.

-12 Edwards A, Elwyn G, Mulley A: Explaining risks: turning numerical data into meaningful pictures. BMJ 2002;324:827-830.

13 Lipkus IM, Hollands JG: The visual communication of risk. J Natl Cancer Inst Monogr 1999;25:149-163.

14 Kühberger A: The influence of framing on risky decisions: a meta-analysis. Organ Behav Hum Decis Process 1998;75:23-55.

15 Gigerenzer G, Edwards A: Simple tools for understanding risks: from innumeracy to insight. BMJ 2003;327:741-744.
16 Gigerenzer G, Hertwig R, van den Broek E, Fasolo B, Katsikopoulos KV: 'A 30\% chance of rain tomorrow': How does the public understand probabilistic weather forecasts? Risk Anal 2005;25:623-629.

17 Koehler JJ: On conveying the probative value of DNA evidence: frequencies, likelihood ratios, and error rates. Univ Colo Law Rev 1996; 67:859-886.

18 Slovic P, Monahan J, MacGregor DG: Violence risk assessment and risk communication: the effects of using actual cases, providing instruction, and employing probability versus frequency formats. Law Hum Behav 2000;24: 271-296.

19 Hamm RM, Smith SL: The accuracy of patients' judgments of disease probability and test sensitivity and specificity. J Fam Pract 1998;47:44-52.

20 Black WC, Nease RF Jr, Tosteson AN: Perceptions of breast cancer risk and screening effectiveness in women younger than 50 years of age. J Natl Cancer Inst 1995;87:720-731.

21 Marteau TM, Saidi G, Goodburn S, Lawton J, Michie S, Bobrow M: Numbers or words? A randomized controlled trial of presenting screen negative results to pregnant women. Prenat Diagn 2000;20:714-718.

22 Hoffrage U, Gigerenzer G: Using natural frequencies to improve diagnostic inferences. Acad Med 1998;73:538-540.

23 Bar-Hillel M: The base-rate fallacy in probability judgments. Acta Psychol 1980;44:211233.

24 Berwick DM, Fineberg HV, Weinstein MC: When doctors meet numbers. Am J Med 1981; 71:991-998.

25 Koehler JJ: The base rate fallacy reconsidered: descriptive, normative, and methodological challenges. Behav Brain Sci 1996;19:1-53.

26 Hoffrage U, Gigerenzer G, Krauss S, Martignon L: Representation facilitates reasoning: what natural frequencies are and what they are not. Cognition 2002;84:343-352.

27 Kurzenhäuser S, Lücking A: Statistical formats in Bayesian inference; in Pohl R (ed): Cognitive Illusions: Fallacies and Biases in Thinking, Judgment, and Memory. Hove, Psychological Press, 2004, pp 61-77.
28 Hertwig R, Hoffrage U: Technology needs psychology: how natural frequencies foster insight in medical and legal experts; in Sedlmeier $\mathrm{P}$, Betsch T (eds): Frequency Processing and Cognition. New York, Oxford University Press, 2002, pp 285-302.

29 Brase G: Which statistical formats facilitate what decisions? The perception and influence of different statistical information formats. $\mathrm{J}$ Behav Decis Making 2002;15:381-401.

30 Kurzenhäuser S, Hoffrage U: Teaching Bayesian reasoning: an evaluation of a classroom tutorial for medical students. Med Teach 2002; 24:516-521.

31 Sedlmeier P, Gigerenzer G: Teaching Bayesian reasoning in less than two hours. J Exp Psychol Gen 2001;130:380-400.

32 Nystroem L, Larsson LG, Wall S, Rutqvist LE, Andersson I, Bjurstam N, Fagerberg G, Frisell J, Tabar L: An overview of the Swedish randomised mammography trials: total mortality pattern and the representivity of the study cohorts. J Med Screen 1996;3:85-87.

- 33 Sarfati D, Howden-Chapman P, Woodward A, Salmond C: Does the frame affect the picture? A study into how attitudes to screening for cancer are affected by the way benefits are expressed. J Med Screen 1998;5:137-140.

34 Weinstein ND: What does it mean to understand a risk? Evaluating risk comprehension. J Natl Cancer Inst Monogr 1999;25:15-20.

35 Marteau TM, Senior V, Sasieni P: Women's understanding of a 'normal smear test result': experimental questionnaire based study. BMJ 2001;322:526-528.

36 Wallsten TS, Budescu DV, Zwick R, Kemp SM: Preference and reasons for communicating probabilistic information in numerical or verbal terms. Bull Psychon Soc 1993;31:135138.

37 Hallowell N, Statham H, Murton F, Green J, Richards M: 'Talking about chance': the presentation of risk information during genetic counseling for breast and ovarian cancer. J Genet Couns 1997;6:269-286.

38 Gurmankin AD, Baron J, Armstrong K: The effect of numerical statements of risk on trust and comfort with hypothetical physician risk communication. Med Decis Making 2004;24: 265-271. 